\title{
BMJ Open Reducing risks associated with medicines and lifestyle in a residential care population with intellectual disabilities: evaluation of a pharmacy review initiative in England
}

\author{
Nick Thayer (D) , ${ }^{1,2}$ Simon White (D) , Jasmeen Islam (D) , ${ }^{3}$ Wesley Jones, ${ }^{2,4}$ \\ Stephanie Kenzie, ${ }^{3}$ Rajni Kullu ${ }^{3}$
}

To cite: Thayer N, White $\mathrm{S}$, Islam J, et al. Reducing risks associated with medicines and lifestyle in a residential care population with intellectual disabilities: evaluation of a pharmacy review initiative in England. BMJ Open 2021;11:e046630. doi:10.1136/ bmjopen-2020-046630

- Prepublication history for this paper is available online. To view these files, please visit the journal online (http://dx.doi. org/10.1136/bmjopen-2020046630).

Received 06 November 2020 Accepted 25 June 2021

Check for updates

(c) Author(s) (or their employer(s)) 2021. Re-use permitted under CC BY-NC. No commercial re-use. See rights and permissions. Published by BMJ.

${ }^{1}$ School of Pharmacy and Bioengineering, Keele University, Newcastle-under-Lyme,

Staffordshire, UK

${ }^{2}$ Community Pharmacy Cheshire and Wirral, Runcorn, UK

${ }^{3}$ Cheshire and Wirral Partnership NHS Foundation Trust, Chester, UK

${ }^{4}$ Boots UK Ltd, Nottingham, UK

Correspondence to

Nick Thayer;

n.m.thayer@keele.ac.uk

\section{ABSTRACT}

Objectives A collaborative service initiative involving community pharmacists and a specialist mental health pharmacist was developed to provide pharmacist reviews for care home residents with intellectual disabilities (IDs). This study aimed to characterise the medicines and lifestyle risk outcomes of the service and determine how these align with national priority issues in ID.

Design Descriptive statistical analysis of routinely collected service delivery data.

Setting Residential care homes in the Wirral, England for people with ID.

Participants 160 residents.

Interventions Pharmacist review of residents' medicines and lifestyle risk factors between November 2019 and May 2020.

Primary and secondary outcome measures Numbers of medicines prescribed, the nature of pharmacists' interventions/recommendations and general practitioner (GP)/psychiatrist acceptance.

Results The 160 residents were prescribed 1207 medicines, $74 \%$ were prescribed $\geq 5$ medicines and 507 interventions/recommendations were made, averaging 3.3 per resident. The highest proportion $(30.4 \%)$ were lifestyle risk related, while changing and stopping medicines accounted for $17.9 \%$ and $12.8 \%$, respectively. Of the recommendations discussed with GPs/psychiatrists, $86 \%$ were accepted. Medicines with anticholinergic properties were prescribed for 115 (72\%) residents, of whom $43(37 \%)$ had a high anticholinergic burden score. Pharmacists recommended anticholinergic discontinuation or dose reduction for $28(24 \%)$ residents. The pharmacists made interventions/recommendations about constipation management for $10 \%$ of residents and about respiratory medicines for 17 (81\%) of the 21 residents with respiratory diagnoses.

Conclusions The findings indicate considerable polypharmacy among the residents and a high level of pharmacists' interventions/recommendations about medicines and lifestyle risk, most of which were accepted by GPs/psychiatrists. This included anticholinergic burden reduction and improving respiratory disease and constipation management, which are national priority

\section{Strengths and limitations of this study}

- This is the first study to evaluate a novel collaborative pharmacy service model, including both community and specialist mental health pharmacists in intellectual disability residential care.

- This study uses routinely collected data that make both the study and future work amenable to replication.

- The study findings relate to one geographical area in England only.

- Pharmacists had limited access to residents' healthcare records; greater access may have reduced or changed some of the recommendations made to general practitioners/psychiatrists.

issues. Wider adoption of collaborative pharmacist review models could have similar benefits for residential populations with ID and potentially reduce pressure on other health services.

\section{INTRODUCTION}

An intellectual disability (ID) is defined as a 'reduced intellectual ability and difficulty with everyday activities which affects someone for their whole life'. ${ }^{1}$ People with ID often have complex care needs, with an increased likelihood of premature death. ${ }^{2}$ This was highlighted in The Learning Disability Mortality Review (LeDeR) Annual Report $2018,{ }^{3}$ which described a disparity in the age of death for people with ID, compared with the general population. The Wirral Joint Strategic Needs Assessment cites individuals with ID are possibly twice as likely to have asthma, 25 times more likely to have epilepsy and have higher levels of mental health problems. ${ }^{4}$ Nationally, the National Health Service (NHS) Long Term Plan (2019) commits to ensuring people with ID get 
better support and to improve the use of psychotropic medication with the nationally established programme Stopping the Over-Medication of People with Learning Disabilities (STOMP) ${ }^{56}$

Within care homes for older people, pharmacist input is known to improve patient outcomes by improving medicines administration accuracy and efficiency and reducing adverse effects of medicines and medicines administration errors. ${ }^{7}$ In particular, patient care is improved through exploration of practical medicines issues such as formulation and dose timing. ${ }^{7}$ More broadly, pharmacist interventions in care homes can improve clinically significant outcomes, such as the number of falls, ${ }^{8}$ deprescribing or identifying safety issues. ${ }^{9}$ Using tools such as STOP-START, studies have demonstrated reductions in polypharmacy, optimisation of patient medicines and cost savings. ${ }^{10}$

Despite the similarity of the setting, there is little evidence of the impact of pharmacist interventions in residential care for people with ID, where medicines are also administered. One small-scale study reported that a high number of interventions were made when a pharmacist conducted medicines reviews with people with ID, of which over $40 \%$ concerned the ordering of repeat prescription medicines. ${ }^{11}$ However, limited detail about these interventions was provided and so determining the pharmaceutical needs of people with ID largely remains a matter of assumption based on known poorer health outcomes. ${ }^{12}$ These assumptions include overprescribing of antipsychotics, ${ }^{5}$ that medicines side effects are not well recognised or managed, ${ }^{13}$ polypharmacy (more pronounced in residential institutions) $)^{14}$ and other issues that affect the population at large such as lifestyle risk factors and suboptimal medicines adherence.

The NHS England work stream-Medicines Optimisation in Care Homes ${ }^{15}$-facilitates and funds pharmacists, based in primary care, to conduct medicines reviews in care homes. In the Wirral, conducting pharmacist reviews for ID care home residents was identified as a gap by the Clinical Commissioning Group (CCG), in line with the NHS Long Term Plan (2019). ${ }^{6}$ Furthermore, data provided by Wirral CCG showed variation in prescribing trends, with the average number of medicines per ID resident varying from 0 to more than 11 across different residential locations. A similar variation was seen in the prescribing of antipsychotics, antidepressants and sedatives. To make best use of local pharmacist resource, a cross-sector, collaborative initiative was designed to coordinate the reviews between community and specialist mental health pharmacists. This involved pharmacists from community pharmacies (both independent and large company) and the local Specialist Mental Health NHS Foundation Trust.

This initiative was commissioned by Wirral CCG to provide a combination of pharmacist reviews and care home wastage audits. The evaluation of the service initiative presented here relates to the pharmacist reviews, and the aim of the evaluation was to characterise the medicines and public health outcomes of the collaborative pharmacist review service for residents with ID and determine how these align with the LeDeR report priorities. ${ }^{3}$

\section{METHODS}

\section{Description of the service initiative intervention}

The collaborative pharmacist review service initiative involved pharmacists reviewing ID care home residents' medicines using a structured review framework and where applicable making interventions or recommendations to the resident's general practitioner (GP) and/or psychiatrist. The framework for the reviews was devised jointly by the lead consultant psychiatrist, the strategic lead pharmacist from the Mental Health Trust and the Local Pharmaceutical Committee (LPC) representatives to align with the 2018 LeDeR report ${ }^{3}$ priorities. This report was central to determining the predicted pharmaceutical care gap in this population because many of the areas for improvement in care link directly to the use or understanding of medicines by patients or carers. The framework guided the pharmacists through consultations, ensuring capture of basic information, before focusing on the key recommendations of the LeDeR report. ${ }^{3}$ These included medicines adherence and burden (particularly the anticholinergic burden), respiratory care, vaccination status, constipation risk, sepsis prevention, dysphagia risk and lifestyle risk issues, especially smoking. Finally, pharmacists were asked to detail actions taken/advice provided, any recommendations made and make referrals, as necessary.

Representatives from the Specialist Mental Health Trust and the LPC provided oversight of the initiative, which included developing an implementation plan with the pharmacists who conducted the reviews and providing updates to the commissioner and the Specialist Mental Health Trust, in line with the governance frameworks. Using anonymised, aggregated data (at ID care home level of analysis) provided by Wirral CCG, the oversight group separated the ID care homes into two groups based on the number of psychotropic medicines known to be prescribed to residents. Pharmacists were aligned to care homes based on anticipated patient need, psychotropic medicine use and existing care relationships established through local medicines supply. Those homes with residents with few or no psychotropic medicines tended to be assigned to community pharmacists. Those with higher psychotropic use tended to be assigned to the specialist mental health pharmacist who, as part of the review, focused on the resident's psychotropic care, comparing care plans from the resident's GP and consultant psychiatrist.

The pharmacists then contacted their assigned care homes directly and arranged opportunities to visit and review residents' medicines. Residential homes varied in size and number of residents, but the maximum occupancy varied from 2 to 29 residents. On arrival, the care homes' prescription ordering, medicines storage and medicines management processes were reviewed, and 
any wastage (and opportunities to reduce) were noted. Subsequently, each resident was asked for consent to a medicines review, unless advised by the staff that this was not in the resident's best interests, in which case a paper review (eg, Medicines Administration Records, home notes, etc) was undertaken instead. Where it was found that a resident had no capacity to consent, and there were no notes detailing a previous assessment, pharmacists were asked to establish a best interest decision in line with General Pharmaceutical Council guidelines and the Mental Capacity Act 2005.

Pharmacists used individual residents' care home records to conduct the reviews. The specialist mental health pharmacist also had access to the care record held by the Specialist Mental Health Trust, if the resident was under the Trust's care and remote access to the local data sharing platform. This provides clinicians outside the GP surgery access to limited clinical patient data, such as recent blood tests, physical health monitoring (eg, blood pressure) and medicines prescribed by the GP. Any urgent queries or concerns to a resident's immediate health were discussed with their GP via telephone, querying current treatment and requesting input or action as appropriate. Where appropriate, the community pharmacists followed the service initiative pathway to triage residents to the specialist mental health pharmacist and/or their GP. Where appropriate, the specialist mental health pharmacist discussed the resident's care with the consultant psychiatrist.

Several assessments were made, dependent on the resident's medicines history. These included a modified Glasgow Antipsychotic Side-effect Scale (GASS) and the online tool Medichec (used to measure the anticholinergic burden), currently used within the Trust's normal practice. Additionally, the validated Medication Adherence Report Scale (MAR-5) ${ }^{16}$ and the COPD Assessment Test (CAT)/Asthma Control Test (ACT), frequently used within community pharmacy practice to assess medicine use. Questions were also asked to confirm the resident's influenza/pneumococcal vaccination and smoking status. Wherever possible, the pharmacists also advised on and influenced the resident's general health and lifestyle risk factors, such as by making referrals to local smoking cessation services, with agreement to do so in the review. Other examples of lifestyle factors included diet and/or constipation risk and management, alcohol, managing blood pressure and exercise, vaccination information and advice on the use of supplements or non-prescribed medication.

Following the review, GP surgeries and psychiatrists were contacted by the pharmacists to arrange a review of their recommendations. As the pharmacists were not prescribers, decisions on accepting recommendations were made by the resident's GP/psychiatrist (after reviewing the resident's full clinical record) in consultation with the pharmacists. Any changes to the resident's repeat medicines were made, requests for blood tests or referrals completed and a record of the review recorded on the GP system.
Details of each review and the outcomes of pharmacists' recommendations were also recorded electronically on PharmOutcomes, a web-based secure clinical service recording platform, commonly used within community pharmacy. The outcomes were categorised by the pharmacist at the point of data entry. The individual pharmacists coordinated their own interventions, ensuring that all outcomes were recorded and followed up with the resident's GP. Regular telephone conference calls between the pharmacists, consultant psychiatrist and the oversight group provided a forum to share learnings and highlight possible trends. This also provided a link between the pharmacists and the lead consultant psychiatrist to discuss in depth the care of individual residents.

\section{Data collection and analysis}

Following institutional ethical approval, an evaluation of the data that were routinely collected by the pharmacists as part of delivering the service was undertaken. This involved downloading data from PharmOutcomes into Microsoft Excel and removing all personally identifiable data. The outcome categories recorded by the pharmacists were ratified by the author NT, based on the free text within the consultation record. The data were then transferred to IBM SPSS Statistics V.24 and subjected to descriptive statistical analysis.

The analysis included identifying and thematically grouping the pharmaceutical care needs of the ID resident population in the study as determined by the interventions/recommendations made by the pharmacists. The data were also assessed against the LeDeR report ${ }^{3}$ priorities of medicine adherence and burden, respiratory care, vaccination status, constipation risk, sepsis prevention and dysphagia risk.

\section{Patient and public involvement}

The study design and outcome measures were informed by national priorities, ${ }^{3}$ which were based on observed health outcomes and the priorities and experiences of people with ID and those who care for them. The study involved routinely collected data that directly concerned these national priorities, and as such, the study was also based on the priorities and experiences of people with ID.

\section{RESULTS}

A total of 160 residents received reviews by pharmacists (76 by community pharmacists and 84 by the specialist mental health pharmacist) between November 2019 and May 2020. The advent of the COVID-19 pandemic meant that the service was paused prematurely. The mean age of the cohort was 56 (SD 13.43), ranging from 24 to 79. Of the 160 residents, $31 \%$ were reported as female and $69 \%$ as male.

The analysis of the data is presented with the characterisation of the nature and number of pharmacists' interventions/recommendations first. This is followed 
Table 1 Number of pharmacists' interventions or recommendations identified by type and by number of the 160 residents for whom at least one intervention/ recommendation of each type was made

\begin{tabular}{|c|c|c|}
\hline $\begin{array}{l}\text { Interventions/ } \\
\text { recommendations made } \\
\text { by the pharmacists }\end{array}$ & $\begin{array}{l}\text { Total number } \\
(\%)\end{array}$ & $\begin{array}{l}\text { Number of unique } \\
\text { residents (\%) }\end{array}$ \\
\hline $\begin{array}{l}\text { Adherence (immediate } \\
\text { change/advice provided to } \\
\text { improve) }\end{array}$ & $25(4.9)$ & $22(13.8)$ \\
\hline $\begin{array}{l}\text { Lifestyle risk related } \\
\text { (including referral for } \\
\text { smoking cessation) }\end{array}$ & $154(30.4)$ & $89(55.6)$ \\
\hline $\begin{array}{l}\text { Recommendation - } \\
\text { change to medication } \\
\text { (form/dose/quantity) }\end{array}$ & $91(17.9)$ & 75 (46.9) \\
\hline $\begin{array}{l}\text { Recommendation - start } \\
\text { medication }\end{array}$ & $25(4.9)$ & $23(14.4)$ \\
\hline $\begin{array}{l}\text { Recommendation - stop } \\
\text { medication or query } \\
\text { medication and stop if no } \\
\text { rationale }\end{array}$ & 65 (12.8) & 49 (30.6) \\
\hline $\begin{array}{l}\text { Referred for later blood } \\
\text { pressure review }\end{array}$ & $11(2.2)$ & $11(6.9)$ \\
\hline Referred onwards & $42(8.3)$ & $33(20.6)$ \\
\hline $\begin{array}{l}\text { Requests for blood } \\
\text { monitoring }\end{array}$ & $43(8.5)$ & $36(22.5)$ \\
\hline $\begin{array}{l}\text { Side effects identified } \\
\text { and advice provided to } \\
\text { manage }\end{array}$ & $12(2.4)$ & $12(7.5)$ \\
\hline Update records & $12(2.4)$ & $12(7.5)$ \\
\hline Other & $27(5.3)$ & $17(10.6)$ \\
\hline Total & 507 & \\
\hline
\end{tabular}

by an analysis of whether the pharmacists' interventions/ recommendations were accepted by the resident's GP or psychiatrist. The analyses of the pharmacists' interventions and recommendations according to the LeDeR report ${ }^{3}$ priorities are presented last.

\section{Analysis of pharmacists' interventions/recommendations}

There were a total of 507 interventions or recommendations made by the pharmacists, averaging 3.3 per resident (median 3.0, SD 1.97). Table 1 shows a breakdown of the interventions and recommendations made by type and the number of residents for whom the pharmacists made at least one intervention or recommendation of that type. In some reviews where multiple medicines required the same recommendation or intervention, the pharmacist made the same intervention/recommendation more than once (eg, recommendations to stop medication or change to improve adherence).

Table 1 also shows that most interventions concerned changes to residents' medicines regimes. There was a total of 65 evidence-based recommendations made for 49 residents to stop (or query and potentially stop) medicines, the most common of which were folic acid (8), procyclidine (7), proton pump inhibitors (7) and antipsychotics
(5). In addition, there were 23 residents for whom the pharmacist recommended that a medicine be started (and 25 individual recommendations), the most common of which concerned laxatives (7), statins (4), vitamin D (2) and bisphosphonates (2).

The pharmacists also commonly made lifestyle risk related interventions and recommendations. For example, of the 157 residents who had their smoking status recorded, 46 (29.3\%) were current smokers and 7 of these agreed to a referral to the local smoking cessation services. Fourteen $(9 \%)$ residents received further information to manage their blood pressure, $3(2 \%)$ received advice on alcohol consumption and 44 (28\%) received additional dietary advice and/or advice on managing constipation.

\section{Acceptance of pharmacists' interventions and recommendations}

Following identification of where interventions were needed, including those requiring prompt attention, the pharmacists discussed their recommendations with the resident's GP/psychiatrist. The recommendations were reviewed and actioned where agreement was reached. Interventions already actioned by the pharmacist directly (most usually related to lifestyle risk, medicines use or adherence advice) were shared with the GP for information only. The onset of the COVID-19 pandemic paused the project without any certainty of continuation. Changing guidance and focusing on essential activities across the NHS meant that the pharmacists were not able to discuss all their recommendations for some residents with the GP.

Of the 160 residents consulted by pharmacists, 168 interventions/recommendations (for 136 residents) were discussed with the GP/psychiatrist. Of these 168 interventions, $144(86 \%)$ were accepted and actioned, while 24 (14\%) were not. Reasons for why GPs did not agree with pharmacists' recommendations were lost to follow-up in some instances due to the COVID-19 pandemic, which precluded further analysis. A summary of the recommendations discussed with GPs/psychiatrist is shown in table 2 .

\section{Analysis of medicines adherence and burden}

The 160 residents were prescribed a total of 1207 medicines, $74 \%$ of residents were prescribed five or more medicines (ie, polypharmacy ${ }^{17}$ ), the median number of medicines per resident was seven and the range was 0-20 medicines. All residents had their medication adherence assessed, using the MAR-5, a primarily self-assessment questionnaire validated across a range of health conditions. ${ }^{16}$ The number of residents who reported their adherence to be 25 out of 25 was 126 (79\%), which broadly tallied with the $25(16 \%)$ consultations where the pharmacist made interventions/ recommendations linked directly to suboptimal medicines adherence.

In their reviews, the pharmacists identified that 115 residents (72\%) were prescribed medicines with anticholinergic 
Table 2 Summary of the acceptance of pharmacists' interventions/recommendations when discussed with the GP/psychiatrist

\begin{tabular}{lccc}
\hline $\begin{array}{l}\text { Interventions/ } \\
\text { recommendations }\end{array}$ & $\begin{array}{l}\text { Agreed - } \\
\text { actioned, } \\
\mathbf{n}(\%)\end{array}$ & $\begin{array}{l}\text { Not } \\
\text { agreed, } \\
\mathbf{n ~ ( \% )}\end{array}$ & Total \\
\hline $\begin{array}{l}\text { Recommendation - } \\
\text { change to medication } \\
\text { (form/dose/quantity })\end{array}$ & $51(89)$ & $6(11)$ & 57 \\
$\begin{array}{l}\text { Recommendation - start } \\
\text { medication }\end{array}$ & $15(68)$ & $7(32)$ & 22 \\
$\begin{array}{l}\text { Recommendation - stop } \\
\text { medication or query } \\
\text { medication and stop if no } \\
\text { rationale }\end{array}$ & $32(86)$ & $5(14)$ & 37 \\
$\begin{array}{l}\text { Requests for blood } \\
\text { monitoring }\end{array}$ & $15(100)$ & $0(0)$ & 15 \\
$\begin{array}{l}\text { Medicines adverse } \\
\text { effects or clinical } \\
\text { complications identified }\end{array}$ & $10(83)$ & $2(17)$ & 12 \\
\hline $\begin{array}{l}\text { Other } \\
\text { Total }\end{array}$ & $21(84)$ & $4(16)$ & 25 \\
\hline
\end{tabular}

${ }^{*}$ Recommended changes to regular repeat prescription medicine quantities to allow easier management of medication by care home/resident.

GP, general practitioner.

properties, of which the most commonly prescribed were procyclidine, clozapine, olanzapine, carbamazepine and diazepam. The Medichec online tool was used to calculate the anticholinergic burden for each resident, ${ }^{18}$ which is summarised in figure 1 , and shows that the scores ranged from 0 to 11, with the mean being 3.3 (SD 2.2). Residents with anticholinergic burden scores higher than 3 were either identified and directly reviewed by or referred to the specialist mental health pharmacist following the service initiative pathway and $43(37 \%)$ residents had scores warranting such referral and review. Of all the residents prescribed medicines with anticholinergic activity (115),

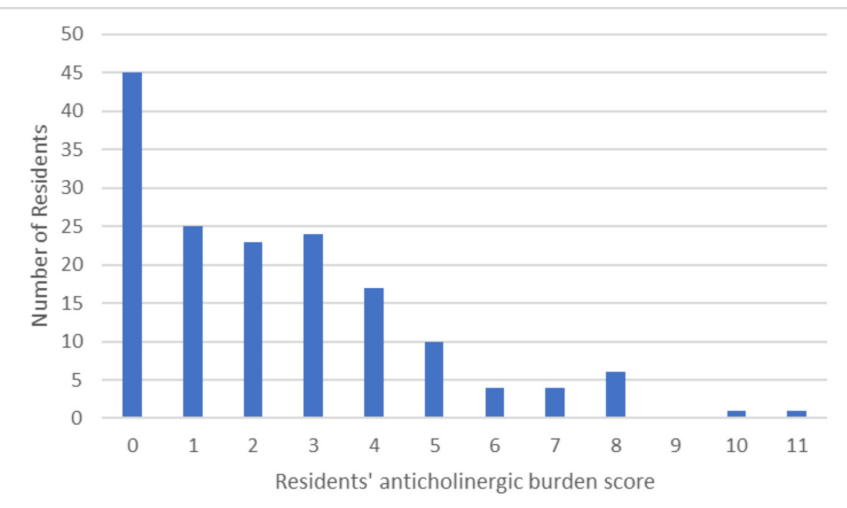

Figure 1 Summary of the anticholinergic burden scores of residents prescribed medicines with anticholinergic properties. it was recommended that medicines with anticholinergic properties be stopped or the dose be reduced for 28 (24\%) residents.

The pharmacists assessed residents' antipsychotic medicines using the GASS ${ }^{19}$ and two of the residents reported a score of 22-42 (moderate side effects), while 89 residents reported a score under 21 (absent/mild side effects). None of the residents reported a score of 43-63 (severe side effects).

\section{Analysis of respiratory care and vaccination status}

Twenty-two of the residents required measurements of their respiratory health as part of the pharmacist's review, of whom $7(4 \%)$ had received ACT and $15(9 \%)$ received a CAT. Of these residents, $14(64 \%)$ were identified as having room to improve their respiratory control. The ACT scores were broken down as: score of 25 asthma under control (one resident), scored 20-24 reasonably well controlled (three residents) and under 20 not controlled (three residents). For the CAT test, three residents scored a CAT score above 20 ('significant room for improvement'), 5 scored 10-20 ('room for improvement') and 7 scored under 10 ('COPD has low impact on health').

Through self-identification or care home records, 22 residents were identified as being diagnosed as having COPD or asthma and 21 (13\% of all the residents) were prescribed a respiratory medicine (ie, an inhaler or montelukast). These residents were prescribed a mean of 2.1 medicines per resident and a range of 1-5 medicines. The pharmacists made interventions/recommendations for 17 of the $21(81 \%)$ residents about their prescribed respiratory medication or their respiratory diagnosis, including medicine formulation or dose changes (two residents), stopping or starting a medicine (two residents), attempts to improve adherence (six residents) and referral to the GP (five residents).

In relation to influenza vaccination status, $116(73 \%)$ of the residents had been vaccinated, 3 residents $(2 \%)$ had declined vaccination, but of the remaining 41 residents, 25 $(16 \%)$ had not been vaccinated and $16(10 \%)$ were unsure of their vaccination status. Where residents' vaccination status could not be confirmed, this meant that the resident was unaware of being vaccinated, no record of vaccination administration could be found by the pharmacist or the care home and the care home were unable to confirm whether the resident had been vaccinated.

\section{Analysis of the risks of constipation, sepsis and dysphagia}

A total of 55 (34\%) residents were prescribed a laxative. For 10 of these residents, the pharmacist had recommended a change to the timing of doses or choice of laxative to improve efficacy, or a change aimed at improving adherence, such as changing to a formulation with a different flavour. In addition, the pharmacists recommended that seven residents be started on a laxative. As such, the pharmacists needed to make an intervention or recommendation for approximately $10 \%$ of the residents about constipation management. 
None of the residents who were reviewed by the pharmacists were identified as having signs or symptoms of sepsis. However, two residential homes were provided with additional written material to provide information to the staff about the signs and symptoms of sepsis.

In relation to the risk of dysphagia, four residents reported difficulties taking their medication due to possible swallowing difficulties, and the pharmacist recommended changes to alternative formulations. Conversely, six residents were prescribed liquid formulations when there was no recorded or known rationale or improvement in adherence and alternate formulations were recommended.

\section{DISCUSSION}

This study found a high level of polypharmacy among residents with ID (three-quarters of residents were taking five or more medicines). Through this service initiative, the pharmacists identified a high number of interventions/recommendations (a median of 3 per resident) that were needed to improve residents' treatment, of which $86 \%$ were accepted by the residents' GP/psychiatrist. Previous reports have already established that people with ID have higher pharmaceutical care needs due to multimorbidity and polypharmacy ${ }^{11}$ and a corresponding reduced life expectancy. ${ }^{13}$ This novel and collaborative pharmacist review service initiative reported here drew on the skill sets of pharmacists from different sectors and was able to address the wide variety of residents' pharmaceutical care needs, which may not have been fully within the competence of pharmacists from a single sector. It is likely that meeting these pharmaceutical care needs had a positive impact on the health of the ID residents.

The findings of this study also highlight the anticholinergic burden experienced by residents, and reducing this burden for $30 \%$ of those residents with high anticholinergic burden scores (ie, a score over 3 ) is another key outcome of the service initiative. There is increasing evidence of medicines with anticholinergic properties being associated with falls, cognitive deterioration, drowsiness, constipation in older people, an increased risk of dementia and higher mortality. ${ }^{20}$ As such, the use of these medicines should be limited in older people. There is also evidence that these medicines are more commonly prescribed to patients with ID and that these patients are more likely to be prescribed anticholinergic medication to treat symptoms of antipsychotic agents. ${ }^{21-23}$ In this study, approximately three-quarters of the residents were prescribed at least one medicine with anticholinergic activity, of whom over a third had a high anticholinergic burden score. This is higher than or at the very highest end of recent estimates of 20\%-50\% of older people in England being prescribed at least one medication with anticholinergic activity, ${ }^{20}$ even though the average age of the residents in this study was only 56. Our findings therefore strongly support the high prevalence of anticholinergic prescribing in the ID population highlighted in the 2018 LeDeR report and confirms the need for service development initiatives such as is reported here to support the work of the NHS England STOMP programme to reduce overmedication in the ID population.

The interventions made by pharmacists in this study also addressed other priority areas of care associated with increased mortality and morbidity in the population with ID, particularly respiratory care where $80 \%$ of residents prescribed respiratory medicines required interventions. It is known that people with ID have an increased incidence of asthma and COPD, and within Wirral, both diagnoses of asthma and COPD are slightly higher than the national average $(7.6 \%$ and $2.6 \%$, respectively). ${ }^{25}$ Respiratory diseases were highlighted in the 2018 LeDeR report as being the highest single cause of death $(19 \%)$ in the general population with ID. ${ }^{26}$ While we found no previous studies of respiratory care specific to people with ID, there is an increasing body of literature on pharmacists' interventions in asthma and COPD management in the general population, which has shown benefits in terms of increased adherence to medicines and reductions in routine hospital visits and other health-related costs. ${ }^{27}{ }^{28}$ Our findings suggest that similar findings are likely in the residential population with ID, but further research is warranted to confirm this.

In this study, the pharmacists also made interventions/recommendations about constipation management, another LeDeR priority area, for $10 \%$ of residents. Effective management of constipation in people with ID is essential as it has a higher prevalence than in the general population and is a common cause of preventable death. ${ }^{3}$ As such, the study findings suggest that this should continue to be an important focus for pharmaceutical care reviews in the residential population with ID.

However, limitations of the study include that the results cannot necessarily be generalised across the country, since the study population were ID care home residents from a single CCG area. Additionally, the interventions were undertaken with restricted access to individual patient records. Greater access to these records may have changed or reduced the need for some of the recommendations to GPs and psychiatrists. Finally, the study focused on specific concerns of medication management that are known and published (eg, in the LeDeR report).

The reviews conducted by the pharmacist followed a broad framework, focusing on national priorities. The reviews themselves did not exclude any area of medicines optimisation and were led by the individual resident's needs. However, the evaluation focuses on the extent to which this pharmacist-led initiative can impact on these national priorities and therefore did not consider the broader implications of other medicinesrelated interventions beyond reporting the number and nature of them. Similarly, this study focuses exclusively on care home residents, rather than people living both 
independently and otherwise in the community. This reflected the current CCG priorities and highlighted health need, but future initiatives would benefit from exploring the requirements of this related population, as well as including them in service pathways.

Nevertheless, the data presented here do suggest benefit for residents with ID, and the introduction of Primary Care Networks in England offers an opportunity for such cross-sector collaborative service models to be developed, which would be expected to contribute to workload pressure reduction for GPs, emergency health services and secondary care services. The findings of this study suggest that commissioners should consider the frequency of reviews within this population group and how to best use the available resource within the pharmacy profession. However, a full evaluation of the benefit for residents with ID and the wider health economy of any larger scale uptake of this service initiative would require an appropriately designed randomised controlled trial, which should include a health economic evaluation. This would likely require a prior feasibility study to identify viable primary and secondary outcomes to determine the effectiveness of the service. The economic evaluation should include clinical and humanistic outcomes in addition to economic outcomes. ${ }^{29}$ Qualitative exploration of the perspectives of community pharmacists', GPs', care home staff and Specialist Mental Health Trust staff, among others, on the outcomes and quality of the service provided might also yield useful insights.

\section{Twitter Nick Thayer @nickthayer99 and Jasmeen Islam @Jasmeenlslam}

Acknowledgements The authors would like to thank Charlotte Dunkley and Bradley Broadhurst for their clinical contribution in conducting reviews. Thanks also go to Phil Elliott at Cheshire and Wirral Partnership NHS Foundation Trust and Alison Williams and Adam Irvine at Community Pharmacy Cheshire \& Wirral for their support in realising the project.

Contributors Conceptualisation: NT, SW and JI. Formal analysis: NT and WJ. Writing - original draft: NT and SW. Writing - review and editing: all authors.

Funding The authors have not declared a specific grant for this research from any funding agency in the public, commercial or not-for-profit sectors.

Competing interests None declared.

Patient and public involvement Patients and/or the public were not involved in the design, or conduct, or reporting, or dissemination plans of this research.

Patient consent for publication Not required.

Ethics approval This study received a favourable ethical opinion from the Faculty of Medicine and Health Sciences Research Ethics Committee at Keele University (ref. MH-200109).

Provenance and peer review Not commissioned; externally peer reviewed.

Data availability statement All data relevant to the study are included in the article or uploaded as supplementary information. No additional data available.

Open access This is an open access article distributed in accordance with the Creative Commons Attribution Non Commercial (CC BY-NC 4.0) license, which permits others to distribute, remix, adapt, build upon this work non-commercially, and license their derivative works on different terms, provided the original work is properly cited, appropriate credit is given, any changes made indicated, and the use is non-commercial. See: http://creativecommons.org/licenses/by-nc/4.0/.

\section{ORCID iDs}

Nick Thayer http://orcid.org/0000-0003-1127-9433
Simon White http://orcid.org/0000-0003-0096-251X

Jasmeen Islam http://orcid.org/0000-0002-8828-5738

\section{REFERENCES}

1 Mencap. Learning disabilities: our definition; 2020 [Accessed 09 Jul 2020].

2 Hollins S, Attard MT, von Fraunhofer N, et al. Mortality in people with learning disability: risks, causes, and death certification findings in London. Dev Med Child Neurol 1998;40:50-6.

3 Noah Fry Centre for Disability Studies. The learning disability mortality review $(\mathrm{LeDe} R$ ) programme, annual report 2018. The University of Bristol, 2019.

4 Wirral Intelligence Service. Joint strategic needs assessment: learning disabilities; 2020 [Accessed 13 Jul 2020].

5 NHS England. Stopping over medication of people with a learning disability, autism or both (STOMP).

6 NHS. The NHS long term plan. 2019.

7 McDerby N, Kosari S, Bail K, et al. The effect of a residential care pharmacist on medication administration practices in aged care: a controlled trial. J Clin Pharm Ther 2019;44:595-602.

8 Zermansky AG, Alldred DP, Petty DR, et al. Clinical medication review by a pharmacist of elderly people living in care homes--randomised controlled trial. Age Ageing 2006;35:586-91.

9 Alves A, Green S, James DH. Deprescribing of medicines in care Homes-A five-year evaluation of primary care pharmacist practices. Pharmacy 2019;7. doi:10.3390/pharmacy7030105. [Epub ahead of print: 03 Aug 2019].

10 Baqir W, Hughes J, Jones T, et al. Impact of medication review, within a shared decision-making framework, on deprescribing in people living in care homes. Eur J Hosp Pharm 2017;24:30-3.

11 Shaw N, Hodson K. Clinical pharmacist medication review of patients with learning disabilities: what types of interventions are made? International Journal of Pharmacy and Practice 2016;24:8-13.

12 Haveman M, Heller T, Lee L, et al. Major health risks in aging persons with intellectual disabilities: an overview of recent studies. J Policy Pract Intellect Disabil 2010;7:59-69. doi:10.1111/j.17411130.2010.00248.x

13 O'Dwyer M, Meštrović A, Henman M. Pharmacists' medicinesrelated interventions for people with intellectual disabilities: a narrative review. Int J Clin Pharm 2015;37:566-78.

14 O'Dwyer M, Peklar J, McCallion P, et al. Factors associated with polypharmacy and excessive polypharmacy in older people with intellectual disability differ from the general population: a cross-sectional observational nationwide study. BMJ Open 2016;6:e010505.

15 NHS England. Medicines optimisation in care homes: programme overview, 2018.

16 Chan AHY, Horne R, Hankins M, et al. The medication adherence report scale: a measurement tool for eliciting patients' reports of nonadherence. Br J Clin Pharmacol 2020;86:1281-8.

17 Masnoon N, Shakib S, Kalisch-Ellett L, et al. What is polypharmacy? A systematic review of definitions. BMC Geriatr 2017;17:230.

18 Bishara D, Harwood D, Sauer J, et al. Anticholinergic effect on cognition (AEC) of drugs commonly used in older people. Int $J$ Geriatr Psychiatry, 2017;32:650-6.

19 Schouby Bock M, Nørgaard Van Achter O, Dines D, et al. Clinical validation of the self-reported Glasgow antipsychotic side-effect scale using the clinician-rated UKU side-effect scale as gold standard reference. J Psychopharmacol 2020;34:820-8.

20 Grossi CM, Richardson K, Savva GM, et al. Increasing prevalence of anticholinergic medication use in older people in England over 20 years: cognitive function and ageing study I and II. BMC Geriatr 2020;20, :267.

21 O'Dwyer M, McCallion P, McCarron M, et al. Medication use and potentially inappropriate prescribing in older adults with intellectual disabilities: a neglected area of research. Ther Adv Drug Saf 2018;9:535-57.

22 O'Dwyer M, Maidment ID, Bennett K, et al. Association of anticholinergic burden with adverse effects in older people with intellectual disabilities: an observational cross-sectional study. $\mathrm{Br} \mathrm{J}$ Psychiatry 2016;209:504-10.

23 De Vreese LP, Mantesso U, De Bastiani E, et al. Anticholinergic burden in adult and elderly people with intellectual disabilities: results from an Italian multicenter cross-sectional study. PLoS One 2018;13, :e0205897.

24 Fox C, Smith T, Maidment I, et al. Effect of medications with anti-cholinergic properties on cognitive function, delirium, physical function and mortality: a systematic review. Age Ageing 2014;43:604-15. 
25 Public Health England. Public health profiles: asthma: QOF prevalence (all ages); 2020 [Accessed 20 Jul 2020].

26 Davis S. Asthma in intellectual disability: are we managing our patients appropriately? Breathe 2016:12:310-7.

27 Zhong H, Ni X-J, Cui M, et al. Evaluation of pharmacist care for patients with chronic obstructive pulmonary disease: a systematic review and meta-analysis. Int J Clin Pharm 2014;36:1230-40.
28 Hesso I, Gebara SN, Kayyali R. Impact of community pharmacists in COPD management: inhalation technique and medication adherence. Respir Med 2016;118:j.rmed.2016.07. 010:22-30.

29 Kozma CM, Reeder CE, Schulz RM. Economic, clinical, and humanistic outcomes: a planning model for pharmacoeconomic research. Clin Ther 1993;15:1121-32. 